\title{
EMPI Promotes the Proliferation and Invasion of Ovarian Cancer Cells Through Activating the MAPK Pathway
}

This article was published in the following Dove Press journal: OncoTargets and Therapy

\author{
Yang Liu \\ Yiling Ding \\ Yanting Nie \\ Mengyuan Yang \\ Department of Obstetrics and \\ Gynecology, Second Xiangya Hospital of \\ Central South University, Changsha \\ 4I0083, People's Republic of China
}

Correspondence: Mengyuan Yang Department of Obstetrics and Gynecology, Second Xiangya Hospital of Central South University, Changsha 410083, People's Republic of China Email yangmengyuan_med@csu.edu.cn
Introduction: Epithelial membrane protein 1 (EMP1), a member of the EMP family, is overexpressed in a large number of tumors and is thought to be a cellular connexin on the cell membrane and is involved in proliferation, invasion, metastasis of tumor cells, and epithelial-mesenchymal transition (EMT). Nevertheless, its biomedical function in ovarian cancer is still unclear.

Methods: EMP1 was detected in ovarian cancer cell lines by whole transcriptome resequencing. The mRNA of EMP1 was examined by qRT-PCR. The relationship between expression of EMP1 and clinical classification, metastasis, and shortened survival time in ovarian cancer specimens was analysed by immunohistochemical (IHC). The mechanism of EMP1 enhanced proliferation and invasion of ovarian cancer cells was determined by siRNA interference, colony formation, migration and invasion experiments, and Western blot.

Results: EMP1 was up-regulated in ovarian cancer cell lines and ovarian cancer tissues in comparison with non-cancerous ovarian specimens. High expression of EMP1 in ovarian cancer specimens was obviously related to high clinical classification, metastasis, and shortened survival time. High expressed EMP1 facilitates cell proliferation, invasion and EMT in ovarian cancer cells. Over-expressed EMP1 increased the protein levels of RAS/ RAF/MAPK/c-JUN.

Conclusion: Over-expressed EMP1 in ovarian cancer promotes tumor cell proliferation, invasion, and EMT by the MAPK signaling pathway.

Keywords: EMP1, ovarian cancer, EMT, MAPK

\section{Introduction}

Recently, Epithelial ovarian cancer (EOC) remains the most common gynaecological malignance, ranking fifth among female worldwide cancer-related deaths. ${ }^{1,2}$ Although a great deal of effort has been devoted to the study of new cytotoxic and targeted drugs and surgery, the overall survival rate of patients is still around $40 \%{ }^{3,4}$ Standard treatment is still surgery, paclitaxel, carboplatin and combined chemotherapy, but recurrence and metastasis were happened in about $80 \%$ of patients. ${ }^{5,6}$ As a result, it is crucial to discover significant biomarkers and to know their cellular and molecular mechanism in regulating the invasion and metastasis of ovarian cancer. ${ }^{7}$

The members of the epithelial membrane protein (EMPs) family, including EMP1, EMP2, and EMP3, have four putative transmembrane domains with approximately 160 amino acid residues. ${ }^{8}$ EMPs are encoded by the peripheral 
myelin protein $22 \mathrm{kDa}$ (PMP22) gene family, and involved in tumor cell migration, growth, and differentiation. ${ }^{9}$ EMP1 is also known as CL-40, tumor-associated membrane protein (TMP), B4B. ${ }^{10}$ The EMP1 protein consists of 157 amino acids and is a glycoprotein containing four highly conserved hydrophobic transmembrane domains localized on the membrane. ${ }^{11}$ Studies have shown that EMP1 plays a crucial part in cell adhesion, proliferation, apoptosis, differentiation, tumor formation and metastasis. ${ }^{12,13} \mathrm{Li}$ et al found that in glioblastoma, highly expressed EMP1 promotes tumor cell proliferation, invasion and metastasis by activating PI3K/AKT/mTOR signaling pathway. ${ }^{14}$ Ogita et al found that EMP1 binding with copine-III promoted the expression of Src, Vav2 and Rac1 in prostate cancer, leading to enhanced migration and metastasis of prostate cancer cells, indicating that EMP1 may take a part in the positive regulation of metastasis. ${ }^{15}$ Boer et al found that EMP1 is elevated in childhood acute lymphoblastic leukemia. ${ }^{16}$ Silencing EMP1 inhibits leukemia cell survival and makes leukemia cells sensitive to prednisolone, which causes loss of migration and adhesion with mesenchymal cells.

As far as we know, there is currently no study of the biological mechanism of EMP1 in ovarian cancer. In prior study, mRNA expression profiles of ovarian cancer cell line and non-neoplastic cell line were checked by RNA sequencing technology (RNA-seq). In differentially expressed genes, EMP1 is one of the genes obviously overexpressed in ovarian cancer cell in comparison with nonneoplastic cell $\left(\log _{2} \mathrm{FC}=3.9\right.$ and 4.5 , see $\underline{\text { Supplementary }}$ Table 1).

In this study, we verified that EMP1 is up-regulated in ovarian cancer tissues in comparison with para-carcinoma tissues, and is closely associated with clinical classification, metastasis, and survival prognosis in patients with ovarian cancer. In addition, it was found that overexpressed EMP1 enhanced proliferation, invasion, and EMT of ovarian cancer cells. In addition, EMP1 activates the expression of RAS/RAF/MAPK/c-JUN in the MAPK pathway. As a result, EMP1 enhances cancer invasion by MAPK pathway in ovarian cancer cells.

\section{Materials and Methods}

\section{Patient Ovarian Cancer and Adjacent}

\section{Tissue Specimens}

Thirty-four ovarian cancer tissues and 34 non-cancerous adjacent tissues were obtained from the same patient in the department of obstetrics and gynecology, Second Xiangya Hospital, Central South University. Half of the tissues were washed with saline and immediately transferred to a $-80^{\circ} \mathrm{C}$ refrigerator or liquid nitrogen for subsequent RT-PCR experiment. The other half of the tissues were fixed in $4 \%$ paraformaldehyde for $24 \mathrm{~h}$, dehydrated with graded ethanol, vitrified by dimethylbenzene, and then embedded in paraffin for immunohistochemistry. All ovarian cancer specimens were pathologically verified between 2017 and 2018, and all patients were not treated prior to diagnosis. In 34 patients with ovarian cancer, the age ranged from 37 to 70 years. The clinical data of ovarian cancer patients are shown in Supplementary Table 1. This research is authorized by the Ethics Committee of the Second Xiangya Hospital of Central South University, and the patient consent was written informed consent, and that this was conducted in accordance with the Declaration of Helsinki.

\section{Cell Culture}

Three human epithelial ovarian cancer cell lines containing 3A0, A2780, and SKOV3 and one non-tumor cell line IOSE80 were applied to detect EMP1. All these cell lines were purchased from cell bank of central south university. These ovarian cancer cells were cultivated in DMEM medium (Hyclone, China) with the addition of $10 \%$ FBS (Hyclone, China) and 1\% penicillin-streptomycin. These cells were cultured in an incubator at $37^{\circ} \mathrm{C}$ and $5 \% \mathrm{CO}_{2}$.

\section{Antibodies Used for IHC and Western Blot}

Rabbit anti-EMP1 antibody (ab230445) was purchased from Abcam. Mouse anti-GAPDH (97166), rabbit antiRAS (3339), rabbit anti-RAF (4432S), rabbit anti-MAPK (4695), and mouse anti-c-JUN (L70B11) were all from Cell Signaling Technology (CST). Horseradish peroxidase (HRP)-labeled anti-mouse (7076) and anti-rabbit (7074) secondary antibodies were all derived from CST.

\section{Real-Time Fluorescent Quantitative PCR (qRT-PCR)}

Fresh tissues were extracted and fragmented with Trizol (Beyotime Biotechnology, Chian) as described. $1 \mu \mathrm{g}$ of RNA was used as a template for the synthesis of complementary DNA using SuperScript IV kit (Invitrogen, China). A total of $15 \mu \mathrm{L}$ volume of qRT-PCR include $1 \mu \mathrm{L}$ of cDNA, $1 \mu \mathrm{L}$ of forward and reverse primer, $12 \mu \mathrm{L}$ of Master Mix (Promega, USA), respectively. 
Finally, the PCR detection system (Thermo, ABI 7500 , USA) was used to detect EMP1 expression for each sample. The primer sequences for EMP1 and GAPDH are as follow:

EMP1 forward 5'- CCCTCATTAAGCTGCCTATCAG $\mathrm{T}-3^{\prime}$;

reverse 5'- CACACAGGTATGCCCTGGTT -3';

GAPDH forward 5'- AGAAGGCTGGGGCTCATTT G-3';

reverse 5'- AGGGGCCATCCCACAGTCTTC-3'.

The relative expression of EMP 1 was gained by $2^{-\Delta \Delta C t}$ equation.

\section{Histopathological Evaluation}

The specimens were embedded in paraffin, sectioned $(4 \mu \mathrm{m})$, roasted at $60^{\circ} \mathrm{C}$ for $4 \mathrm{~h}$, and overnight at $37^{\circ} \mathrm{C}$. Dewaxing with xylene, gradient alcohol hydration, incubation of EMP1 primary antibody, goat anti-rabbit secondary antibody labeled with horseradish peroxidase, and finally staining with DAB (Beyotime, P0203, China). The results of EMP1 staining were assessed by pathologists. The immunostaining intensities were 0 (negative), 1 (weak positive), 2 (medium positive), and 3 (strongly positive).

\section{Western Blot Assay}

After the cells were treated, they were lysed by adding RIPA lysate (Beyotime, P0013C, China), and the lysate was collected and centrifuged at $12,000 \mathrm{rpm}$ for $20 \mathrm{~min}$. Protein concentration was measured using a BCA kit (Beyotime, P0012, China). $30 \mu \mathrm{g} / \mathrm{lane}$ of protein was loaded on a $10 \%$ SDS-PAGE and then transferred to a PVDF membrane (invitrogen, USA) by electrotransfer. After blocking for $2 \mathrm{~h}$ with 5\% skim milk, the membrane was incubated for one overnight, and the HRP-labeled secondary antibody was incubated for $2 \mathrm{~h}$ 3and detected by a chemiluminescence imager (Sagecreation, China).

\section{Lentivirus Transfection}

A lentiviral vector with high expression of EMP1 was

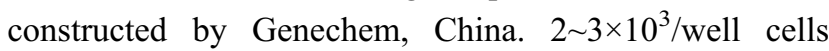
were cultured in 96-well plates until the cell confluence was $20-30 \%$. Add virus and infection enhancement solution, continue to culture for $12 \mathrm{hrs,} \mathrm{change} \mathrm{cell} \mathrm{culture}$ medium and continue to culture for $48 \sim 72 \mathrm{hrs}$.

\section{siRNA Interference}

siRNA1, siRNA2, siRNA3 of EMP1 were designed and synthesized by Genechem Company. When the confluence of ovarian cancer cells in the 6-well plate reached $50 \%$, siRNA1, siRNA2, siRNA3 and NC (100 nM) of EMP1 were transfected with Lipo8000 (Beyotime, C0533, China), respectively. Then incubated for $48 \mathrm{~h}$, and the expression level of EMP1 was evaluated by Western blot. siRNA1 guide 5'-AAUAGCAUAAUAACAGUAGCG-3' passenger 5'-CUACUGUUAUUAUGCUAUUUG-3' siRNA2 guide 5'- UACAGUUUUUCCAAAGACCUA-3' passenger 5'- GGUCUUUGGAAAAACUGUACC-3' siRNA3 guide 5' - UCACGAUUCGCAUAAUGACUA-3' passenger 5'- GUCAUUAUGCGAAUCGUGAUG-3'

\section{Cell Counting Kit-8 (CCK8) Assay}

$2 \times 10^{3} /$ well cells were cultured in 96 -well plate. $10 \mu \mathrm{L}$ of CCK8 (Beyotime, C0038, China) liquid was added to each well, and incubation was continued for $2 \mathrm{~h}$ in a cell culture incubator, and then the absorbance was measured at $450 \mathrm{~nm}$ with a microplate detector (Perkin Elmer, USA). To examine the activation of MAPK on cell proliferation caused by over-expressed EMP1, ovarian cancer cells were firstly treated with arglabin $(20 \mathrm{ng} / \mathrm{mL}$ ) (Sigma, SML1570, USA) for $1 \mathrm{~h}$ prior to cell viability detection.

\section{Colony Formation Experiment}

$500 /$ well cells were cultured in 6-well plates, respectively. After $10 \mathrm{~d}$, the cells were fixed with $4 \%$ paraformaldehyde for $10 \mathrm{~min}$, stained with $0.5 \%$ crystal violet, counted and imaged with an optical microscope.

\section{Migration and Invasion Experiments}

Scratch healing experiments were used to detect the migration ability of EMP1 over-expressed cells. The cells were cultured in a 6-well plate to sub-fusion, and then FBS-free medium was used in each plate. Linear scratches were made with sterile plastic microtube heads. Images were captured at the beginning and periodically during cell migration to close the wound, and images were compared to determine the rate of cell migration.

The invasive ability of the cells was examined using a matrix-coated chamber (Corning, USA). Ovarian cancer cells were counted to ensure a cell concentration of $2.5 \times$ $10^{5} / \mathrm{mL}$. The chamber was inserted into a 24-well plate (700 $\mu \mathrm{L} /$ well) containing DMEM medium containing $20 \%$ FBS. $200 \mu \mathrm{L}\left(2.5 \times 10^{5} / \mathrm{mL}\right)$ of the above cells were inoculated on a transwell plate and incubated for $20 \mathrm{~h}$. Gently wipe the uninjured cells from the upper chamber with a damp cotton swab. The invasive cells in the filter were fixed with $100 \%$ methanol for $10 \mathrm{~min}$, and stained with 
$0.1 \%$ crystal violet (Beyotime, C0121, China) for $20 \mathrm{~min}$. Under the microscope, five random parts of each chamber were calculated.

\section{Statistical Analysis}

The experiment in this study was repeated three times. Statistical analysis was proceeded with SPSS 18.0 software (SPSS, USA). The relationship between EMP1 expression and clinical features in patients with ovarian cancer was evaluated via Chisquare test. In our study, the Kaplan Meier method and the Log-rank test were used to analyze overall survival (OS), progression-free survival (PFS), and distal non-metastatic survival (DMFS). The two sets of data were compared using Student's $t$-test. The difference was statistically significant at $\mathrm{P}<0.05$ $(* \mathrm{P}<0.05 ; * * \mathrm{P}<0.01 ; * * * \mathrm{P}<0.001)$.

\section{Results}

\section{High Expression of EMPI in Ovarian Cancer Tissues and Cells}

EMP1 mRNA in 34 ovarian cancer tissues and 34 noncancerous ovarian epithelial tissues were detected through qRT-PCR. It was found that EMP1 was obviously increased in tumor tissues $(\mathrm{P}<0.01)$ (Figure 1A). EMP1 protein in ovarian cancer epithelial cells such as A2780 and SKOV3 and non-tumor cell line IOSE80 were detected by Western blot. The results showed that EMP1 protein expression levels were relatively low in IOSE80 cells compared to ovarian cancer cells (Figure 1B). Moreover, EMP1 were significantly higher in SKOV3 cells with high metastatic ability compared to $3 \mathrm{~A} 0$ and A2780 cells with low metastatic ability (Figure 1B).

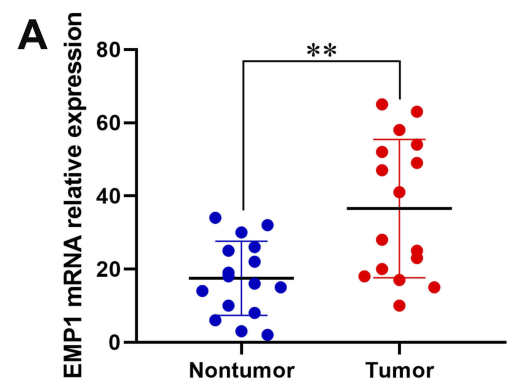

C

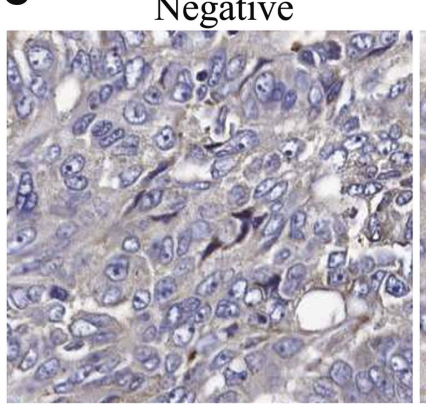

case 1

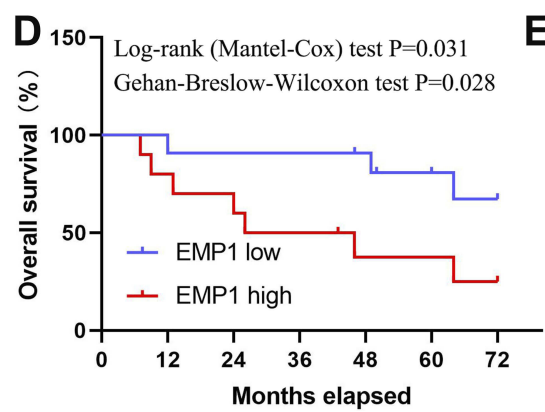

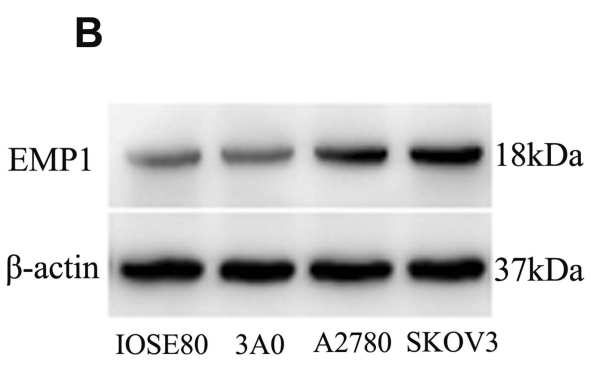

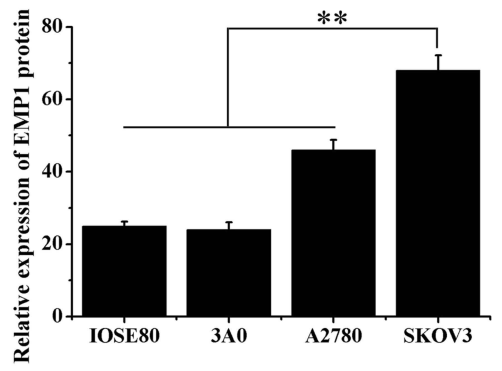

Weak Positve

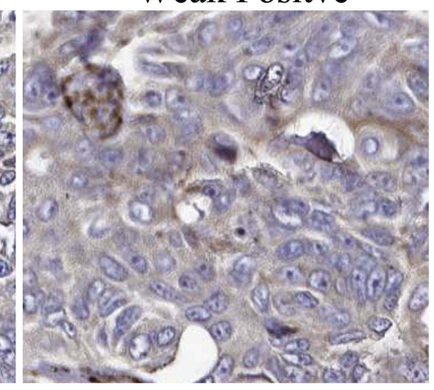

case 2

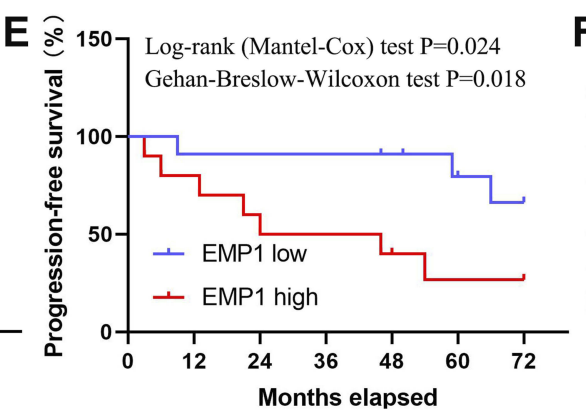

Moderate Positive

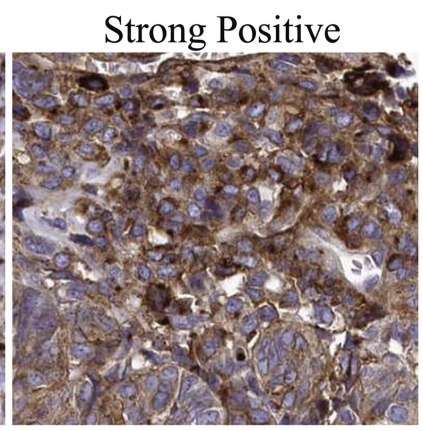

case 4

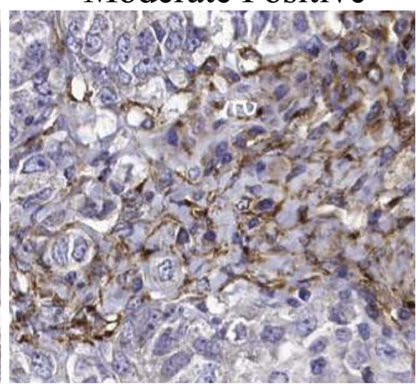

case 3

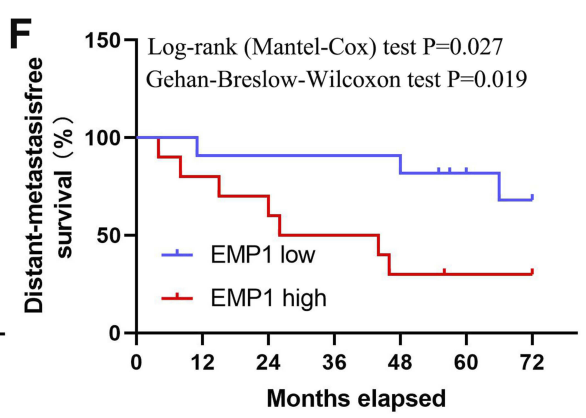

Figure I High expressed EMPI in ovarian cancer associates with poor prognosis. (A) The expression of EMPI mRNA was detected by qRT-PCR. (B) The expression level of EMPI in ovarian tumor cell lines (3A0, A2780, and SKOV3) and the normal cell IOSE80 was detected by Western blot. (C) Representative images of EMPI staining in ovarian tumor patients. Overall survival (OS) (D), progression-free survival (PFS) (E), and distant-metastasis-free survival (DMFS) (F) in ovarian cancer patients. $* * P<0.01$. 


\section{Over-Expressed EMPI Protein Is Related to Disease Progression and Distant Metastasis of Ovarian Cancer}

In order to understand the clinical significance of EMP1 in ovarian cancer patients, 34 paraffin-embedded ovarian cancer tissue specimens were evaluated by IHC. The IHC grades of EMP1 in ovarian cancer specimens ranged from 0 to 3. Representative pictures of ovarian cancer tissues with different classification are shown in Figure 1C. Among them, 15 cases $(42.9 \%)$ had high expression of EMP1 (staining index $>1$ ) in ovarian cancer tissues. As shown in Supplementary Table 2, the relationship between EMP1 expression level and clinical characteristics was assessed by chi-square test. It was shown that increased EMP1 was obviously associated with large tumor volume, lymph node metastasis, advanced clinical stage, and distant metastasis at diagnosis. There was no significant correlation between EMP1 expression and other clinicopathological characteristics. To investigate the prognostic prediction of EMP1 protein in patients with ovarian cancer. It was found that patients with lower EMP1 expression had higher overall survival (OS), progression-free survival (PFS), and no distant metastasis survival (DMFS) (Figure 1D-F). The above data showed that EMP1 took part in the progression of ovarian cancer.

\section{EMPI Promotes Proliferation of Ovarian Cancer Cells}

$3 \mathrm{~A} 0$ and A2780 cells were transfected with EMP1 expressed lentivirus, and the expression EMP1 was verified through Western blot (Figure 2A). For cell viability assay, overexpressed EMP1 obviously promoted the proliferation of $3 \mathrm{~A} 0$ and A2780 cells (Figure 2A). In order to further study the function of EMP1 in ovarian cancer cells, we designed three specific siRNAs against EMP1 to silence EMP1 in SKOV3 cells, respectively. Cell viability assay indicated that knockdown of EMP1 obviously decreased the viability of SKOV3 cells (Figure 2B). In addition, in the colony formation experiments, the number of cell colonies formed by EMP1 overexpressing cells was obviously higher than that of the control cells (Figure 2C). The above results show that EMP1 can promote the proliferation of ovarian cancer cells.

\section{Over-Expressed EMPI Facilitates Migration and Invasion of Ovarian Cancer in vitro}

On account of the connection between EMP1 and ovarian cancer metastasis is supported by clinical evidence, the effect of EMP1 on ovarian cancer cell migration and invasion were studied. In the cell wound healing experiments, the mobility of 3A0-EMP1 and A2780-EMP1 cells was obviously higher than that of the control group (Figure 3A). In the transwell experiment, the number of $3 \mathrm{~A} 0$ and A2780 cells with high expression of EMP1 increased significantly $(\mathrm{P}<0.01$, Figure $3 \mathrm{~B}$ ), while the number of cells invaded by EMP1 knocked out from SKOV3 was 341. Reduced to 94, 122 and 98, respectively (Figure 3C). The above results indicate that EMP1 enhances migration and invasion of ovarian cancer in vitro.

\section{EMPI Enhanced EMT in Ovarian Cancer Cells}

As far as we know, EMT is the most important step in cancer metastasis. ${ }^{17,18}$ In order to elucidate the effect of EMP1 on the metastasis of ovarian cancer cells in vitro, whether EMP1 is involved in EMT of ovarian cancer cells was evaluated by the following experiments. Overexpressed EMP1 could trigger EMT, which showed the expression of mesenchymal marker molecules such as $\mathrm{N}$-cadherin, vimentin and $\beta$-catenin were increased, and the expression of E-cadherin was decreased in 3A0 and A2780 cells by Western blot analysis (Figure 3D). And the opposite result was found in EMP1 knockout SKOV3 cells (Figure 3E). The above results indicate that EMP1 could induce EMT in ovarian cancer cells.

\section{EMPI Enhances Proliferation and Invasion of Ovarian Cancer Cells Through MAPK} Signaling Pathway

Since the MAPK pathway participates in tumor cell proliferation and metastasis and is regularly activated in a large number of cancers including ovarian cancer, ${ }^{19,20}$ Western blot was used to detect the relation between EMP1 and key substrates of the MAPK signaling pathway. In EMP1 knockout SKOV3 cells, RAS/RAF/MAPK/ c-JUN levels were down-regulated (Figure 4A). The results showed that EMP1 activates the MAPK pathway in ovarian cancer cells.

For further research the significance of the MAPK signaling pathway in proliferation and invasion of ovarian cancer, we treated 3A0-EMP1 and A2780-EMP1 cells with the RAS inhibitor (arglabin). The results indicate that arglabin treatment inhibits the ability of EMP1 to promote cell growth (Figure 4B and C). The above results indicate that EMP1 promotes proliferation and invasion in 
A
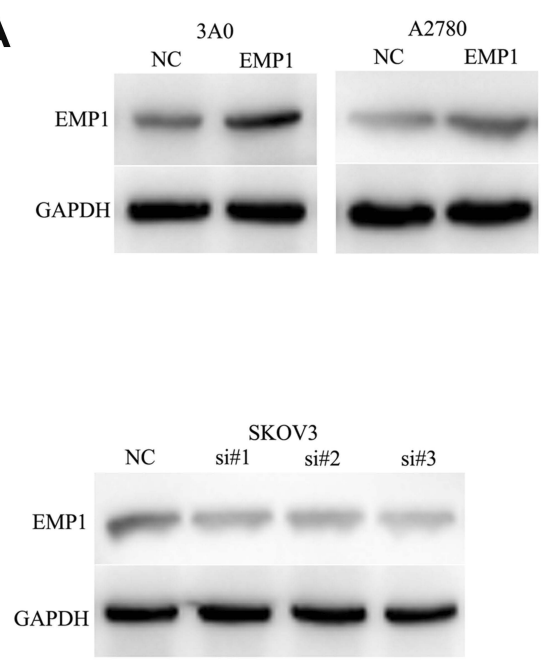
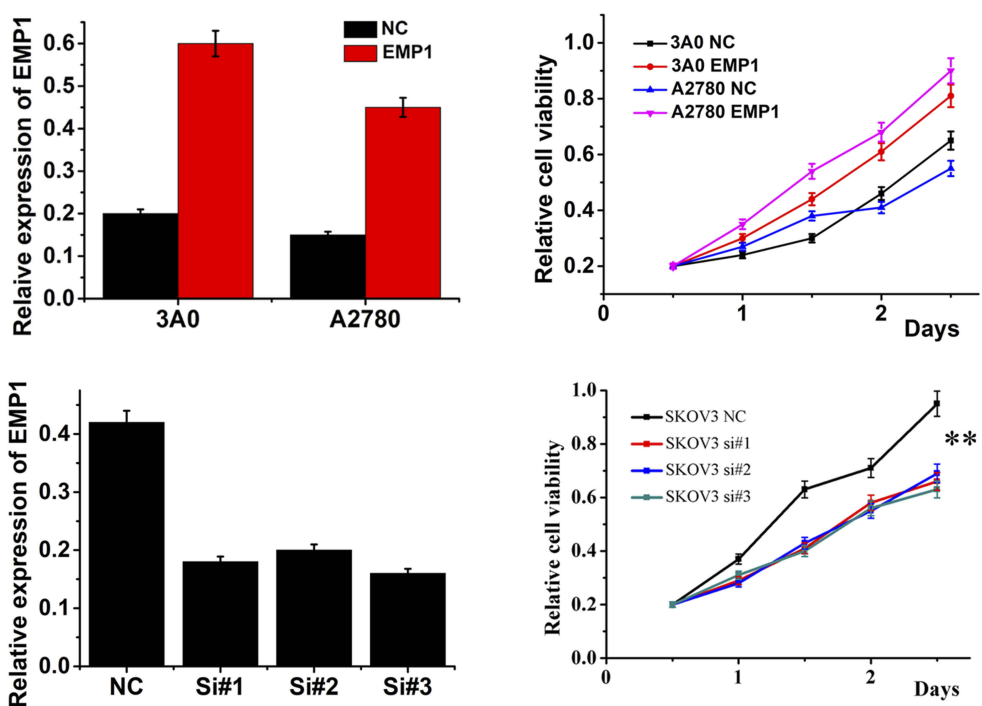
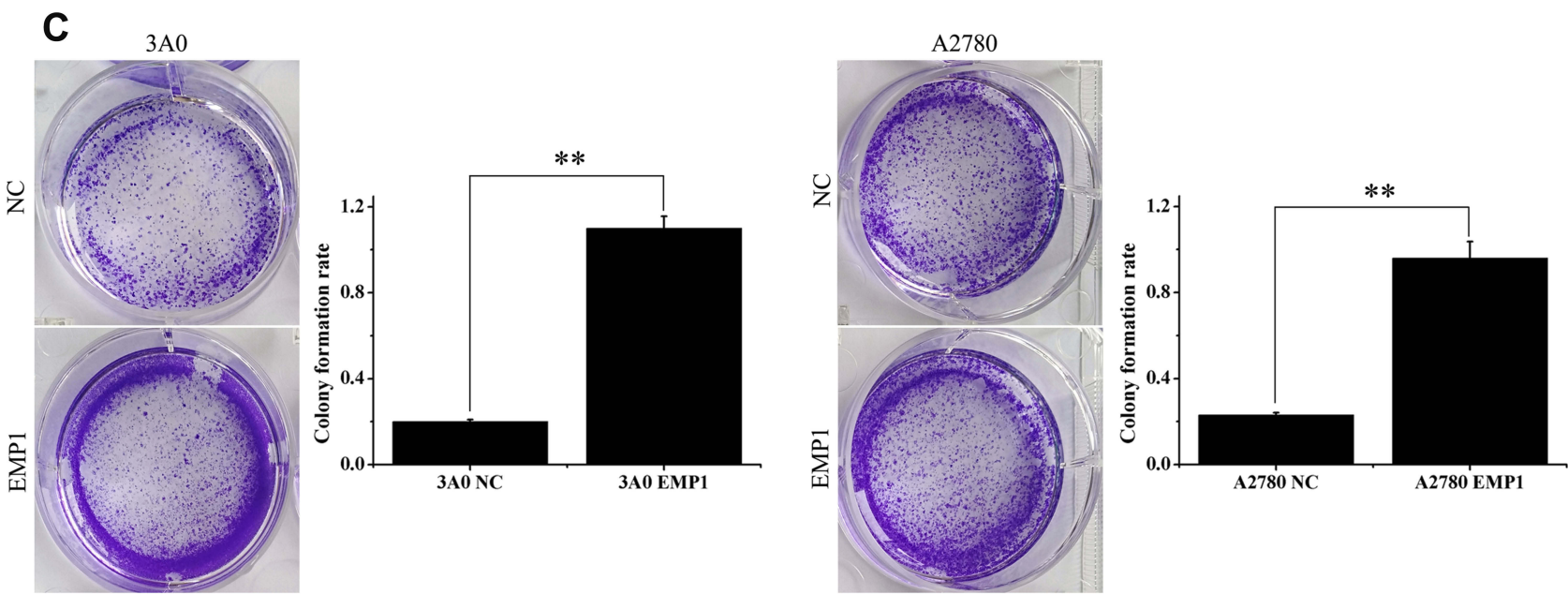

Figure 2 EMPI promotes ovarian cancer cells growth. (A) The expression of EMPI in $3 \mathrm{~A} 0$ and A2780 cells transfected with EMPI expressed lentivirus were detected by Western blot. Cell viability indicated that EMPI could obviously enhance proliferation of 3A0-EMPI and A2780-EMPI cells. (B) Knockdown of EMPI in SKOV3 was verified by Western blot and CCK-8 assays. (C) The colonies were obviously higher in the 3A0-EMPI and A2780-EMPI cells. **P $<0.01$.

ovarian cancer cell depending on the MAPK signaling pathway.

\section{Discussion}

In our prior studies, gene expression profiles of ovarian cancer cell lines A2780 and SKOV3 and a permanent ovarian epithelial cell line IOSE80, were identified by RNA-seq using Illumina Hiseq 2500 sequencing platform to identify transcriptional abnormalities that cause ovarian cancer (unpublished). Compared with IOSE80 cells, 578 and 695 mRNA expressions were different in A2780 and SKOV3 cells, respectively $(p<0.05)$. EMP1 gene was one of the most up-regulated genes, and its expression in A2780 and SKOV3 was significantly higher than IOSE80 $(\operatorname{logFC}=3.9$ and 4.5, see Supplementary Table 1). Furthermore, quantitative analysis by RT-PCR showed that EMP1 mRNA was overexpressed in ovarian cancer tissues than non-cancerous ovarian tissues. EMP1 expression in ovarian cancer cells and tumor tissues was detected by IHC staining. EMP1 protein in SKOV3 cells with high metastatic potential was much higher than that in $3 \mathrm{~A} 0$ and A2780 cells with low metastatic ability. Based on the relation analysis between EMP1 and the clinicopathological characteristics of ovarian cancer, over-expressed EMP1 was obviously positively correlated with tumor size, lymph node metastasis, distant metastasis and clinical stage of ovarian cancer. Significantly, the peritoneal metastasis rate was up to $38.2 \%$ $(13 / 34)$ in the EMP1 high-expression group, while only $14.7 \%(5 / 34)$ in the EMP1 low-expression group. In addition, kaplan-meier and log-rank survival analysis confirmed 
A
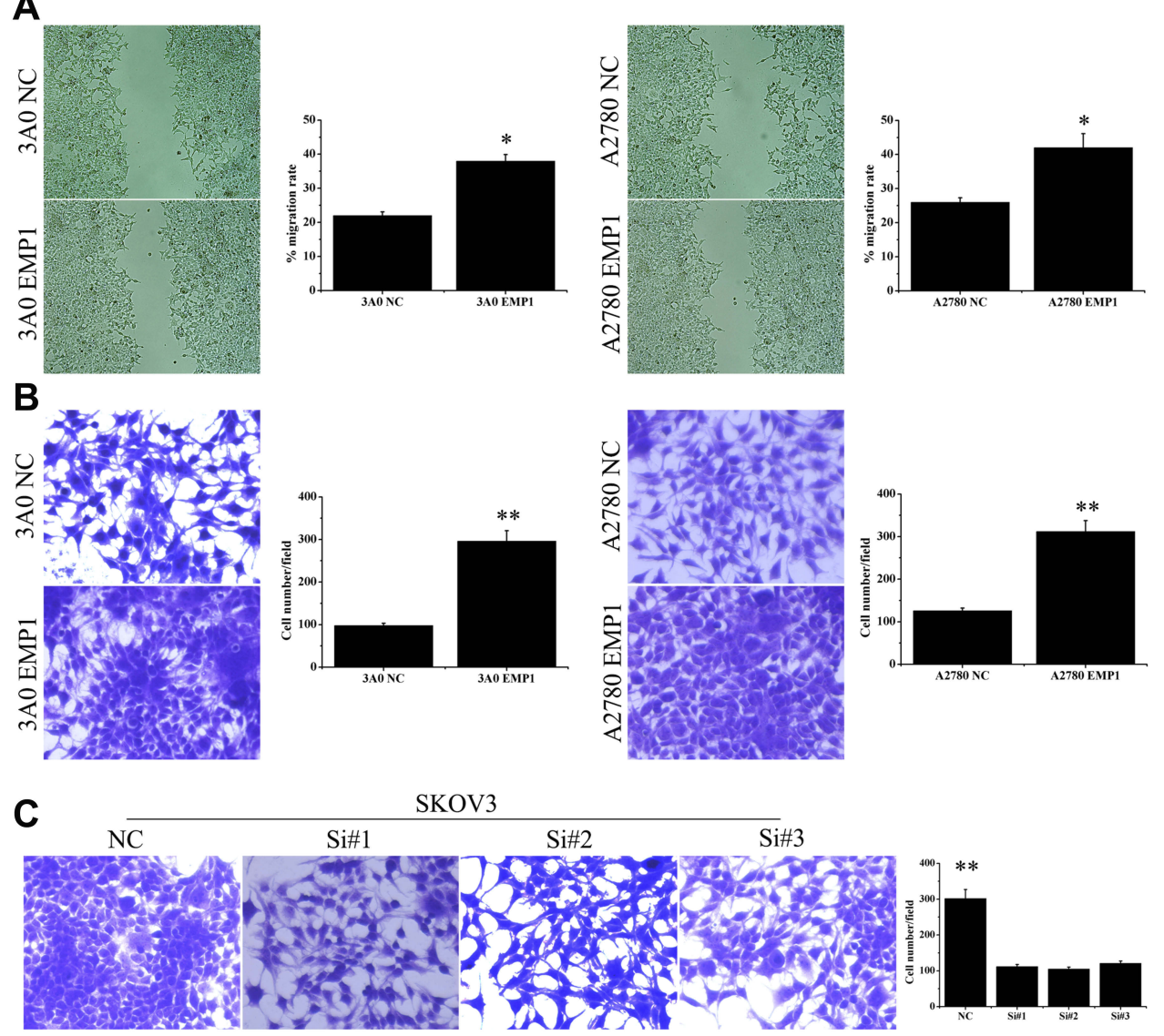

D

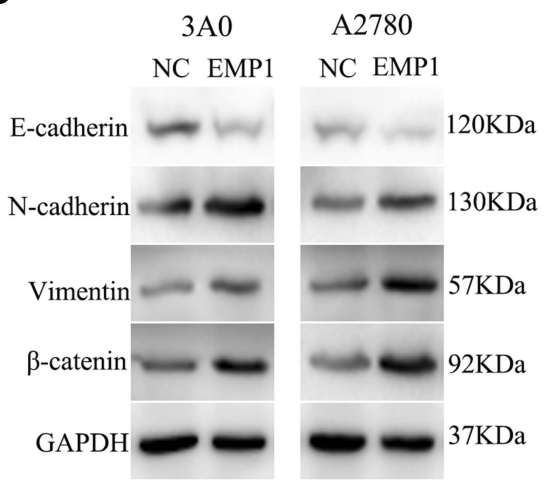

E

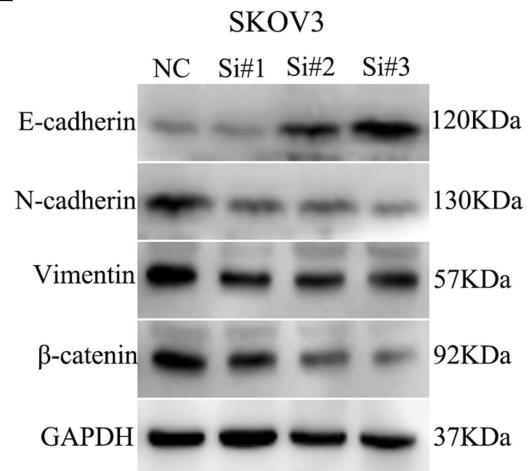

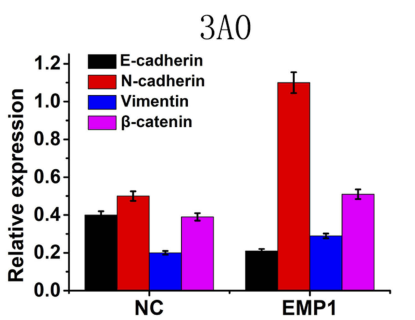
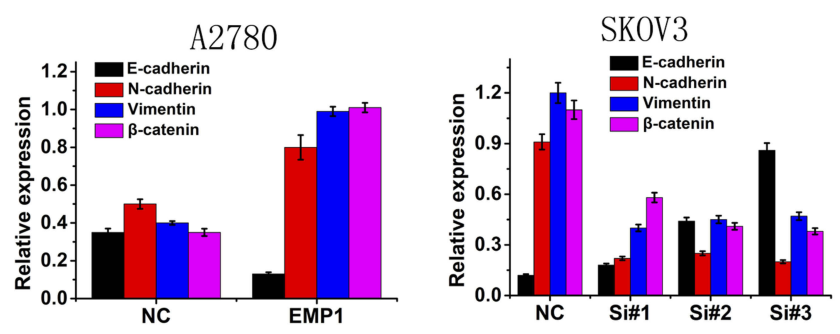

Figure 3 EMPI enhances ovarian cancer cell migration, invasion and EMT. (A) Up-regulated EMPI cells showed stronger migration rate than that of control. (B) The ability of ovarian cancer cell invasion was evaluated by Matrigel invasion chambers. (C) Silencing expression of EMPI in SKOV3 decreased the invaded cells. (D) The expression of epithelial makers and mesenchymal markers in EMPI over-expressed 3A0 and A2780 cells. (E) The expression of epithelial makers and mesenchymal markers in EMPI knockdown cells. $* \mathrm{P}<0.05 ; * * \mathrm{P}<0.01$. 

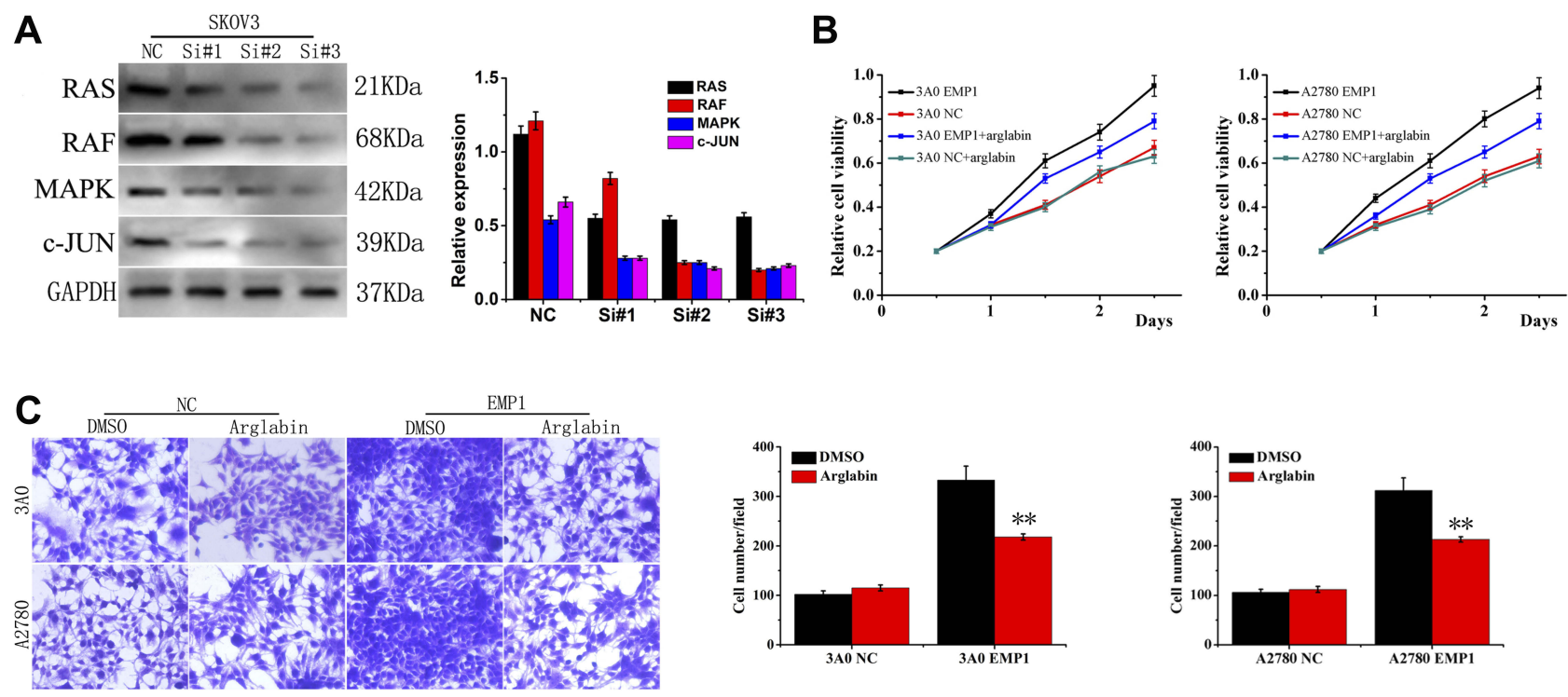

Figure 4 EMPI promotes MAPK signal pathway in ovarian cancer cells. (A) Expression of RAS/RAF/MAPK/c-JUN was reduced in EMPI knockdown SKOV3 cell. Cell viability $(\mathbf{B})$ and invasive abilities $(\mathbf{C})$ of $3 \mathrm{~A} 0$ and $\mathrm{A} 2780$ cells were assessed by $\mathrm{CCK} 8$ and transwell assay after pre-treatment with or without $20 \mathrm{nM}$ of arglabin. $* * \mathrm{P}<0.0 \mathrm{I}$.

the relationship between EMP1 overexpression and poor clinical outcomes (OS, PFS, and DMFS).

For cellular effect, high expressed EMP1 enhanced proliferation, colony formation, migration and invasion of ovarian cancer cells. ${ }^{21,22}$ High-expressed EMP1 upregulated the expression of $\mathrm{N}$-cadherin, vimentin and $\beta$ catenin, and down-regulated E-cadherin. On the contrary, the down-regulation of EMP1 reduced the invasive ability of ovarian cancer cells SKOV3 and inhibited the EMT phenotype. The in vitro and clinical evidence indicate the up-expressed EMP1 enhanced proliferation and invasiveness of ovarian cancer.

MAPK signaling pathway plays an important role in adjusting cell proliferation and metastasis. ${ }^{23}$ MAPK can transfer extracellular stimulation signals to cells and their nuclei, and plays an important role in adjusting cell proliferation, apoptosis, autophagy and migration, and is considered as a hot target in tumor treatment. $^{24,25}$ At present, a large number of studies have been conducted to find inhibitors targeting various links of the MAPK pathway to cut off the signal transduction pathway to inhibit tumors. ${ }^{26}$ In this study, abnormal activation of ovarian cancer in RAS/RAF/MAPK/ c-JUN was found, which was positively correlated with EMP1. When EMP1 was knocked down, the RAS/RAF/ MAPK/c-JUN pathway was also inhibited, and the tumor proliferation was reduced. In addition, RAS inhibitor arglabin eliminated the effect of EMP1 overexpression on the growth and invasion in $3 \mathrm{~A} 0$ and A2780 cells. ${ }^{27}$
Together, these findings support the role of EMP1 in promoting tumor proliferation and invasion by activating the MAPK signaling pathway in ovarian cancer cells.

\section{Conclusion}

Our study is the first to demonstrate that EMP1 is upregulated in ovarian cancer, leading to proliferation, migration, invasiveness and EMT of ovarian cancer cells. Furthermore, EMP1 was found to promote proliferation and invasion of ovarian cancer cells through RAS/RAF/MAPK/ c-JUN signaling pathway. EMP1 is considered as a biomarker and therapeutic target for ovarian cancer prognosis.

\section{Disclosure}

The authors report no conflicts of interest in this work.

\section{References}

1. Huang T, Tworoger SS, Willett WC, et al. Associations of early life and adulthood adiposity with risk of epithelial ovarian cancer. Ann Oncol. 2019;2(30):303-309. doi:10.1093/annonc/mdy546

2. Tewari KS, Burger RA, Enserro D, et al. Final overall survival of a randomized trial of bevacizumab for primary treatment of ovarian cancer. J Clin Oncol. 2019;26(37):2317-2328. doi:10.1200/JCO.19. 01009

3. Peres LC, Cushing-haugen KL, Köbel M, et al. Invasive epithelial ovarian cancer survival by histotype and disease stage. J Natl Cancer Inst. 2019;1(111):60-68. doi:10.1093/jnci/djy071

4. Kawakami E, Tabata J, Yanaihara N, et al. Application of artificial intelligence for preoperative diagnostic and prognostic prediction in epithelial ovarian cancer based on blood biomarkers. Clin Cancer Res. 2019;10(25):3006-3015. doi:10.1158/1078-0432.CCR-18-3378 
5. Marth C, Reimer D, Zeimet AG. Front-line therapy of advanced epithelial ovarian cancer: standard treatment. Ann Oncol. 2017;28: viii36-viii39. doi:10.1093/annonc/mdx450

6. Buechel M, Herzog TJ, Westin SN, et al. Treatment of patients with recurrent epithelial ovarian cancer for whom platinum is still an option. Ann Oncol. 2019;5(30):721-732. doi:10.1093/annonc/mdz104

7. Yang Y, Wu L, Shu X, et al. Genetic data from nearly 63,000 women of european descent predicts DNA methylation biomarkers and epithelial ovarian cancer risk. Cancer Res. 2019;3(79):505-517. doi:10.1158/0008-5472.CAN-18-2726

8. Ahmat Amin MKB, Shimizu A, Ogita H. The pivotal roles of the epithelial membrane protein family in cancer invasiveness and metastasis. Cancers. 2019;11(11):E1620. doi:10.3390/cancers 11111620

9. Fu M, Maresh EL, Soslow RA, et al. Epithelial membrane protein-2 is a novel therapeutic target in ovarian cancer. Clin Cancer Res. 2010;15(16):3954-3963. doi:10.1158/1078-0432.CCR-10-0368

10. Busch M, Klein S, Große-kreul J, et al. p53, miR-34a and EMP1-newly identified targets of TFF3 signaling in Y79 retinoblastoma cells. Int J Mol Sci. 2019;20(17):E4129. doi:10.3390/ijms20174129

11. Wang YW, Cheng HL, Ding YR, et al. EMP1, EMP 2, and EMP3 as novel therapeutic targets in human cancer. Biochim Biophys Acta Rev Cancer. 2017;1(1868):199-211. doi:10.1016/j.bbcan.2017.04.004

12. Durgan J, Tao G, Walters MS, et al. SOS1 and Ras regulate epithelial tight junction formation in the human airway through EMP1. EMBO Rep. 2015;1(16):87-96. doi:10.15252/embr.201439218

13. Wang J, Li X, Wu H, et al. EMP1 regulates cell proliferation, migration, and stemness in gliomas through PI3K-AKT signaling and CD44. J Cell Biochem. 2019;10(120):17142-17150. doi:10.1002/jcb.28974

14. Miao L, Jiang Z, Wang $\mathrm{J}$, et al. Epithelial membrane protein 1 promotes glioblastoma progression through the PI3K/AKT/mTOR signaling pathway. Oncol Rep. 2019;2(42):605-614. doi:10.3892/ or.2019.7204

15. Ahmat Amin MKB, Shimizu A, Zankov DP, et al. Epithelial membrane protein 1 promotes tumor metastasis by enhancing cell migration via copine-III and Rac1. Oncogene. 2018;40(37):5416-5434. doi:10.1038/s41388-018-0286-0

16. Ariës IM, Jerchel IS, van den Dungen RE, et al. EMP1, a novel poor prognostic factor in pediatric leukemia regulates prednisolone resistance, cell proliferation, migration and adhesion. Leukemia. 2014;9 (28):1828-1837. doi:10.1038/leu.2014.80
17. Prakash V, Carson BB, Feenstra JM, et al. Ribosome biogenesis during cell cycle arrest fuels EMT in development and disease. Nat Commun. 2019;1(10):2110. doi:10.1038/s41467-019-10100-8

18. Aiello NM, Kang Y. Context-dependent EMT programs in cancer metastasis. J Exp Med. 2019;5(216):1016-1026. doi:10.1084/jem.20 181827

19. Ma W, Xu Z, Wang Y, et al. A positive feedback loop of SLP2 activates MAPK signaling pathway to promote gastric cancer progression. Theranostics. 2018;20(8):5744-5757. doi:10.7150/thno. 28898

20. Rotow JK, Gui P, Wu W, et al. Co-occurring alterations in the RAS-MAPK pathway limit response to MET inhibitor treatment in MET exon 14 skipping mutation-positive lung cancer. Clin Cancer Res. 2019. doi:10.1158/1078-0432.CCR-19-1667

21. Liu C, Wei X, Li F, et al. The prognostic value of epithelial membrane protein 1 (EMP-1) in patients with laryngeal carcinoma. Med Sci Monit. 2017;23:3795-3800. doi:10.12659/msm.901161

22. Demirag GG, Kefeli M, Kemal Y, et al. Epithelial membrane protein 1 expression in ovarian serous tumors. Oncol Lett. 2016;3(11):21 40-2144. doi:10.3892/ol.2016.4202

23. Berger MD, Stintzing S, Heinemann V, et al. Impact of genetic variations in the MAPK signaling pathway on outcome in metastatic colorectal cancer patients treated with first-line FOLFIRI and bevacizumab: data from FIRE-3 and TRIBE trials. Ann Oncol. 2017;11 (28):2780-2785. doi:10.1093/annonc/mdx412

24. Xiao C, Li M, Huang Q, et al. SPAG9 promotes prostate cancer proliferation and metastasis via MAPK signaling pathway. Am $J$ Transl Res. 2019;8(11):5249-5260.

25. Zhen AX, Hyun YJ, Piao MJ, et al. Eckol inhibits particulate matter 2.5-induced skin keratinocyte damage via MAPK signaling pathway. Mar Drugs. 2019;8(17):E444. doi:10.3390/md17080444

26. Chen Q, Liu J, Wang W, et al. Sini decoction ameliorates sepsis-induced acute lung injury via regulating ACE2-Ang (1-7)Mas axis and inhibiting the MAPK signaling pathway. Biomed Pharmacother. 2019;115:108971. doi:10.1016/j.biopha.2019.108971

27. Lone SH, Bhat KA, Khuroo MA. Arglabin: from isolation to antitumor evaluation. Chem Biol Interact. 2015;240:180-198. doi:10.10 16/j.cbi.2015.08.015
OncoTargets and Therapy

\section{Publish your work in this journal}

OncoTargets and Therapy is an international, peer-reviewed, open access journal focusing on the pathological basis of all cancers, potential targets for therapy and treatment protocols employed to improve the management of cancer patients. The journal also focuses on the impact of management programs and new therapeutic

Submit your manuscript here: https://www.dovepress.com/oncotargets-and-therapy-journa agents and protocols on patient perspectives such as quality of life, adherence and satisfaction. The manuscript management system is completely online and includes a very quick and fair peer-review system, which is all easy to use. Visit http://www.dovepress.com/ testimonials.php to read real quotes from published authors. 